\title{
Rastreio cognitivo em pacientes com acidente vascular cerebral: um estudo transversal
}

\author{
Cognitive screening in patients with stroke: a cross-sectional study
}

Ana Amália Torres Souza Gandour Dantas', Sara Viviane de Souza Torres', Izabel Myckilane Alves de Farias', Suzane Beatriz Calixto de Lira Sant'Ana', Tania Fernandes Campos'

\section{RESUMO}

Objetivo: $O$ estudo objetivou-se a fazer um rastreio cognitivo nos pacientes com acidente vascular cerebral (AVC), a fim de determinar pontos de corte de acordo com a idade, escolaridade e grau de comprometimento neurológico. Métodos: Foi realizado um estudo transversal no qual participaram 109 pacientes ambulatoriais, sendo 61 homens, com média da idade de 59 anos ( \pm 11), média do tempo de escolaridade de 5 anos $( \pm 4)$ e do tempo de sequela de 16 meses $( \pm 14)$. Os pacientes foram avaliados por meio do Miniexame do Estado Mental (MEEM) e pela National Institute of Health Stroke Scale. Os dados foram analisados pela regressão linear múltipla (stepwise forward). Resultados: Verificou-se que as variáveis grau de comprometimento neurológico, idade e escolaridade contribuíram significativamente para o valor global do MEEM e explicaram a variância do estado cognitivo $\left(R^{2}\right.$ ajustado $\left.=0,24\right)$. Cada aumento do comprometimento neurológico representou diminuição de 0,456 no escore do MEEM. Quanto maior a idade, ocorreu uma diminuição de 0,202 no MEEM, e à medida que diminui o tempo de escolaridade, houve uma diminuição de 0,190 no MEEM. Os pontos de corte variaram de 14 a 22 de acordo com o grau de comprometimento neurológico, idade e escolaridade. Conclusão: Os resultados apontaram que, por meio do rastreio positivo de déficit cognitivo, foram encontrados pontos de corte associados ao comprometimento neurológico, necessitando também serem ajustados pela idade e escolaridade, sugerindo que essas associações sejam preferencialmente levadas em consideração na planificação da reabilitação neuropsicológica dos pacientes com AVC.

\section{ABSTRACT}

Objective: The aim of this study was to make a cognitive screening in patients with stroke in order to determine cutoff points according to age, education and degree of neurological impairment. Methods: A cross-sectional study was conducted. The study included 109 outpatients (61 men), mean age 59 years $( \pm 11), 5$ years of schooling $( \pm 4)$ and 16 months sequel time $( \pm 14)$. Patients were assessed by the Mini-Mental State Examination (MMSE) and the National Institute of Health Stroke Scale. Data were analyzed by multiple linear regression (stepwise forward). Results: It was found that the variable degree of neurological impairment, age and education contributed significantly to the overall value of the MMSE

1 Universidade Federal do Rio Grande do Norte (UFRN), Departamento de Fisioterapia, Programa de Diagnóstico e Intervenção no Acidente Vascular Cerebral (PRODIAVC).

\section{Recebido em \\ $6 / 3 / 2013$ \\ Aprovado em}

$5 / 5 / 2014$ 


\section{Keywords}

Stroke, Mini-Mental State

Examination, cognition. and explained variance in cognitive status (adjusted $R^{2}=0.24$ ). Each increase of neurological impairment represented a decrease of 0.456 in MMSE score. The greater the age, there was a decrease of 0.202. As the years of education decreased there was a decrease in MMSE score of 0.190 . The cutoffs ranged 14-22 according to the degree of neurological impairment, age and education. Conclusion: The results showed that through positive screening for cognitive impairment cutoffs associated with neurological impairment were found, need to be adjusted for age and education, suggesting that these associations are preferably taken into consideration in planning the neuropsychological rehabilitation of stroke patients.

\section{INTRODUÇÃO}

O acidente vascular cerebral (AVC) é uma patologia definida como uma disfunção neurológica causada por uma anormalidade na circulação cerebral. Os pacientes apresentam manifestações clínicas diversas, tais como comprometimentos na função sensório-motora, cognição, percepção, linguagem e deficiências visuais, que limitam as atividades no ambiente social, profissional e familiar ${ }^{1-3}$.

A literatura mostra que os déficits cognitivos em pacientes com AVC dependem da combinação de três fatores. Primeiro, a localização da lesão. Segundo, a distribuição das disfunções neuronais que determinam a velocidade do processamento cognitivo, memória e redução das funções executivas. Terceiro, o nível de comprometimento cognitivo depende de fatores como idade, sexo, comorbidades, entre outros ${ }^{4}$.

Estima-se que $35 \%$ dos pacientes apresentam algum tipo de comprometimento cognitivo nos três primeiros meses após o AVC e que continuam apresentando até três anos depois ${ }^{5-7}$. Aproximadamente, $10 \%$ dos pacientes após o primeiro AVC desenvolvem demência e 30\% dos indivíduos após AVC recorrentes também ${ }^{8,9}$. Outro estudo apontou que mais da metade dos pacientes após um AVC sofre de déficit cognitivo como problemas na memória e bradipsiquismo, e na fase aguda esses comprometimentos podem passar despercebidos ${ }^{10}$.

Déficits cognitivos podem influenciar na recuperação do status funcional do paciente durante a reabilitação, fazendo com que esse processo se estenda, dificultando a adesão ao tratamento e comprometendo a qualidade de vida dos enfermos ${ }^{4,11}$. Dessa forma, é de fundamental importância fazer o rastreamento dos déficits cognitivos o mais precocemente possível, para isso, os instrumentos de avaliação devem ser sensíveis e fidedignos.

O Miniexame do Estado Mental (MEEM) é o instrumento mais utilizado para rastreamento cognitivo no mundo. O ponto de corte mais frequentemente utilizado para indicar comprometimento cognitivo é o 24. Alguns autores sugerem 25 para aumentar a sensibilidade para demência leve. O ponto de corte é frequentemente ajustado pelo nível educacional. Alguns autores já sugeriram que o ponto de corte 24 mostrou-se excelente para pessoas com escolaridade acima de nove anos, enquanto o ponto de corte 17 foi ótimo para aqueles com menor escolaridade ${ }^{12}$.

No Brasil, os estudos também divergem quanto ao ponto de corte para cada idade e escolaridade. Um estudo realizado com o objetivo de investigar o melhor ponto de corte do MEEM para rastrear déficit cognitivo em uma amostra de idosos com e sem demência verificou que, entre os diferentes níveis de escolaridade avaliados (analfabetos, primário, ginásio, colegial e faculdade), apenas o grupo sem escolaridade formal diferia dos demais quando o efeito da idade era levado em consideração e revelou que o ponto de corte 19/20 (caso/não caso) foi o mais adequado para o rastreamento de déficit cognitivo em idosos sem escolaridade e o 23/24 para idosos com alguma instrução escolar (sensibilidade de $78 \%$ e especificidade de $75 \%)^{13}$.

Avaliando a literatura pesquisada, não foi encontrado nenhum estudo que procurasse identificar pontos de corte específicos para pacientes com AVC, dessa forma o objetivo deste estudo foi fazer um rastreio cognitivo nos pacientes com AVC, a fim de verificar pontos de corte adequados para esses pacientes. A hipótese do estudo é de que é necessário utilizar pontos de corte diferenciados para pacientes com AVC de acordo com a idade, a escolaridade e o grau de comprometimento neurológico.

\section{MÉTODOS}

\section{Amostra}

O estudo realizado foi do tipo transversal. A amostra foi constituída por 109 pacientes ambulatoriais com diagnóstico de primeiro episódio de AVC, registrados em Serviços de Fisioterapia, sendo 48 mulheres e 61 homens, que foram avaliados consecutivamente quando não eram excluídos pelos critérios adotados: lesão cerebral recorrente, transtornos cognitivos graves, afasia e uso de ansiolíticos, antidepressivos ou neurolépticos. 


\section{Procedimentos}

A pesquisa foi aprovada pelo Comitê de Ética em Pesquisa da Universidade Federal do Rio Grande do Norte, por meio do parecer no 193/2006, seguindo os padrões éticos de acordo com a Resolução no 196/96 do Conselho Nacional de Saúde. Os participantes foram informados sobre os procedimentos da pesquisa e assinaram o Termo de Consentimento Livre e Esclarecido (TCLE).

Os pacientes chegavam ao Serviço de Fisioterapia com o diagnóstico médico de AVC e com exames clínicos que comprovavam a doença. Em seguida, um pesquisador fisioterapeuta fazia a avaliação do grau de comprometimento neurológico por meio da National Institute of Health Stroke Scale (NIHSS), que é composta por 11 domínios (nível de consciência, movimentos oculares, campo visual, movimentos faciais, função motora do membro superior e do membro inferior, ataxia de membros, sensibilidade, linguagem, disartria, negligência espacial), sendo pontuados com escores que variam de 0 a 4. Com a soma da pontuação de cada item, é obtido um escore total, e quanto maior for o seu valor, maior é o comprometimento neurológico. Considera-se que escores de 0 a 6 indicam comprometimento neurológico leve, de 7 a 16, comprometimento moderado e de 17 a 30, comprometimento neurológico grave ${ }^{14}$.

O pesquisador também realizava a avaliação cognitiva pelo MEEM, composto por sete categorias que avaliam funções cognitivas específicas como a orientação temporal e espacial, memória imediata, atenção e cálculo, memória com evocação tardia, linguagem e capacidade construtiva visual. O exame gradua o desempenho cognitivo em uma escala de 0 a 30 pontos; pontuação abaixo de 24 indica disfunção cognitiva ${ }^{13}$.

As avaliações neurológica e cognitiva deram respaldo para determinar que os pacientes estavam aptos a entender e dar seu consentimento informado para participar do estudo. Ao assinar o TCLE, os pacientes tinham a garantia de proteção conforme as normas da bioética.

\section{Análise estatística}

Os dados foram analisados utilizando-se o programa SPSS 15.0 (Statistical Package for the Social Science), atribuindo-se o nível de significância de 5\% para todos os testes estatísticos. Após a realização do teste Kolmogorov-Smirnov, foi encontrada distribuição normal nos escores do MEEM, por isso modelos explicativos foram construídos por meio da análise de regressão linear múltipla, a fim de identificar as variáveis preditoras do déficit cognitivo dos pacientes. Antes, foram analisadas por meio do teste de correlação de Pearson as correlações entre as variáveis demográficas (sexo, idade e escolaridade) e clínicas (tempo de sequela e grau de comprometimento neurológico) como variáveis independentes, com o valor global do MEEM (variável dependente), para es- tabelecer a ordem de entrada das variáveis no modelo de regressão. Após a análise univariada, foram selecionadas as variáveis com $p$ valores $<0,10$ (a fim de verificar se na análise multivariada as variáveis fossem significativas com $p<0,05$ ), sendo acrescentadas uma a uma no modelo de regressão em ordem decrescente do coeficiente de correlação (stepwise forward). Os valores do percentil 50 foram calculados para estabelecer pontos de corte de acordo com a idade, escolaridade e grau de comprometimento neurológico.

\section{RESULTADOS}

Por meio da análise descritiva, foi verificado que a amostra apresentava média e desvio-padrão da idade de $59 \pm 11$ anos, da escolaridade de $5 \pm 4$ anos, do tempo de sequela de $16 \pm 14$ meses e dos escores do grau de comprometimento neurológico de $5 \pm 4$.

A análise de correlação realizada entre o sexo, idade, escolaridade, tempo de sequela e grau de comprometimento neurológico com os escores do MEEM apontou que o sexo e o tempo de sequela não apresentaram um $p<0,10$, sendo excluídos da análise de regressão (Tabela 1). As demais variáveis foram acrescentadas uma a uma, observando-se o comportamento do modelo à medida que era introduzida cada variável. Ao final da análise de regressão, verificou-se que as variáveis grau de comprometimento neurológico, idade e escolaridade contribuíram significativamente para o valor global do MEEM, sendo o modelo 3 o que melhor explicou a variância do estado cognitivo ( $R^{2}$ ajustado $=0,24-$ Tabela 2). Nesse sentido, cada aumento do comprometimento neurológico representa uma diminuição de 0,456 no escore do MEEM. Da mesma forma, quanto maior a idade, ocorre uma diminuição de 0,202 no MEEM, e à medida que diminui o tempo de escolaridade, há uma diminuição de 0,190 no MEEM.

Em função dos resultados da análise de regressão, foram calculados os percentis 50 para determinar os pontos de corte do MEEM. Os pontos de corte variaram de 14 a 22 de acordo com o grau de comprometimento neurológico, idade e escolaridade (Tabela 3).

Tabela 1. Coeficientes de correlação entre o MEEM e as variáveis demográficas e clínicas

\begin{tabular}{lcc}
\hline & \multicolumn{2}{c}{ MEEM } \\
\cline { 2 - 3 } & Coeficientes $(r)$ & p valor \\
\hline Sexo & 0,087 & 0,369 \\
Idade & $-0,175$ & 0,069 \\
Escolaridade & 0,164 & 0,089 \\
Tempo de sequela & $-0,057$ & 0,555 \\
Grau de comprometimento neurológico & $-0,376$ & 0,0001 \\
\hline
\end{tabular}


Tabela 2. Modelos de regressão linear múltipla (stepwise forward), considerando grau de comprometimento neurológico, idade e escolaridade como variáveis independentes e o escore global do Miniexame do Estado Mental (MEEM) como variável dependente Valores de $R^{2}$ ajustado, $\beta \pm$ EP (erro-padrão), intervalo de confiança de $95 \%$ e p valor

\begin{tabular}{|c|c|c|c|c|}
\hline & $R^{2}$ ajustado & $\beta \pm E P$ & IC de 95\% & $p$ \\
\hline \multicolumn{5}{|l|}{ Modelo 1} \\
\hline Grau de comprometimento neurológico & 0,14 & $-0,376 \pm 0,124$ & $-0,767$ a $-0,274$ & 0,0001 \\
\hline \multicolumn{5}{|l|}{ Modelo 2} \\
\hline Grau de comprometimento neurológico & 0,21 & $-0,428 \pm 0,123$ & $-0,837 a-0,351$ & 0,0001 \\
\hline Idade & & $-0,261 \pm 0,042$ & $-0,205$ a $-0,041$ & 0,004 \\
\hline Modelo 3 & 0,24 & & & \\
\hline Grau de comprometimento neurológico & & $-0,456 \pm 0,122$ & $-0,875$ a $-0,391$ & 0,0001 \\
\hline Idade & & $-0,202 \pm 0,043$ & $-0,180$ a $-0,009$ & 0,030 \\
\hline Escolaridade & & $0,190 \pm 0,119$ & 0,010 a 0,484 & 0,041 \\
\hline
\end{tabular}

Tabela 3. Percentis dos escores do Miniexame do Estado Mental (MEEM) de acordo com o grau de comprometimento neurológico (leve e grave), a idade e a escolaridade

\begin{tabular}{lcc}
\hline & $\begin{array}{c}\text { Leve } \\
\text { Percentil 50 }\end{array}$ & $\begin{array}{c}\text { Grave } \\
\text { Percentil 50 }\end{array}$ \\
\hline Escolarizados & 22 & \\
$\geq 60$ anos & & 19 \\
Não escolarizados & 19 & 14 \\
$\geq 60$ anos & & \\
Escolarizados & 23 & 21 \\
$<60$ anos & & \\
Não escolarizados & 18 & 16 \\
$<60$ anos & 18 & \\
\hline
\end{tabular}

\section{DISCUSSÃO}

No presente estudo, a análise de regressão apontou que os escores do MEEM podem variar primariamente de acordo com o grau de comprometimento neurológico, ou seja, o comprometimento neurológico deve ser levado em consideração antes de se estabelecer um ponto de corte para déficit cognitivo nos pacientes com AVC. Os escores podem ser estabelecidos diferentemente para pacientes com grau leve e grave, e à medida que o grau de comprometimento neurológico melhora, o ponto de corte no MEEM aumenta. Em seguida, por ordem de associação encontrada, a idade foi identificada como um fator de ajuste necessário, sugerindo que o aumento da idade leva a uma diminuição do ponto de corte do MEEM. O terceiro fator relacionado foi a escolaridade, indicando que à medida que diminui o tempo de escolaridade, há uma diminuição no ponto de corte do MEEM.

A literatura é ampla em estudos que consideram que o MEEM deve ser ajustado pela idade e escolaridade. Em geral, a idade avançada é um importante preditor de déficit cognitivo depois do AVC e de demência na fase crônica do
AVC 1,15,16. Além disso, a literatura aponta que valores específicos para diferentes escolaridades são necessários ao interpretar resultados individuais do MEEM em populações de baixo nível educacional para reduzir a taxa de falsos-positi$\operatorname{vos}^{12,13,17}$.

Estudos anteriores mostram que os pacientes com diferentes tipos de patologias podem ter desempenho diferenciado no MEEM, dependendo, por sua vez, das lesões apresentadas. Indivíduos parkinsonianos podem apresentar mais dificuldades no item de atenção e cálculo, no desenho ou na repetição; por outro lado, pacientes com a doença de Alzheimer inicial poderão apresentar somente déficit mais evidente na recordação das palavras, e indivíduos com quadros de confusão mental provavelmente terão maior comprometimento nos itens de orientação ${ }^{18}$.

Em pacientes com AVC, o grau de comprometimento neurológico pode variar dependendo da fase de acometimento. Déficits cognitivos específicos podem ser mais comuns em indivíduos mais idosos na fase aguda do AVC. Por exemplo, vários estudos têm mostrado que a negligência hemiespacial é mais comum em indivíduos mais velhos do que nos mais jovens que tiveram AVC ${ }^{19,20}$. A afasia depois do AVC parece ser mais comum em indivíduos mais velhos com $3 \%$ de aumento do risco por ano adicionado de idade ${ }^{21}$. Outro importante fator na avaliação de déficits cognitivos na fase aguda é o efeito de medicamentos ${ }^{22}$.

Muitos déficits cognitivos continuam além do período subagudo. Em um estudo, cerca de 83\% indivíduos com déficits iniciais na memória verbal e 78\% indivíduos com déficits na construção visuoespacial e memória visual mostraram recuperação somente aos seis meses ${ }^{23}$. Outro estudo mostrou que 54\% dos pacientes ainda apresentavam déficits na atenção um ano após o AVC, mas poucos tinham déficits na função executiva, linguagem e memória de longo prazo comparado com a fase aguda ${ }^{24}$. Além disso, alguns indivíduos podem ter progressão dos déficits cognitivos até desenvolverem demência ${ }^{25}$. Todas essas evidências indicam 
que, dependendo da fase do AVC, o comprometimento cognitivo pode variar; nesse sentido, é possível que o ponto de corte em testes de rastreio também deva ser ajustado.

No presente estudo os pontos de corte do MEEM para o AVC foram definidos da seguinte forma: para pacientes com grau de comprometimento neurológico leve - ponto de corte 22 para pacientes com idade $\geq 60$ anos escolarizados e 19 para não escolarizados; ponto de corte 23 para pacientes com idade < 60 anos escolarizados e 18 para não escolarizados; para pacientes com grau de comprometimento neurológico grave - ponto de corte 19 para pacientes com idade $\geq$ 60 anos escolarizados e 14 para não escolarizados; ponto de corte 21 para pacientes com idade $<60$ anos escolarizados e 16 para não escolarizados. Nesse sentido, os pontos de corte mais baixos foram encontrados em pacientes com grau de comprometimento neurológico grave, comparando-se a idade e a escolaridade de pacientes com grau de comprometimento neurológico leve. Todos os pontos de corte encontrados foram abaixo do ponto de corte 24 , que é o estabelecido na literatura para rastreio de déficits cognitivos ${ }^{12}$.

Esses pontos de corte encontrados podem permitir um rastreio cognitivo mais adequado, e os déficits cognitivos podem ser classificados de acordo a literatura em quatro grupos: grupo 1, déficit severo (escore de 0 a 10); grupo 2, déficit moderado (de 11 a 20); grupo 3, déficit leve (de 21 a 24), e grupo 4, sem déficit (de 25 a 30)4. Entretanto, é importante ressaltar que, apesar de o MEEM ser é um instrumento que tem sido utilizado em ambientes clínicos para detecção de declínio cognitivo, para o seguimento de quadros demenciais e no monitoramento de resposta ao tratamento ${ }^{26}$, os seus resultados não podem permitir um diagnóstico de comprometimento cognitivo definitivo e preciso, fazendose necessário encaminhamento do paciente para avaliação neuropsicológica mais ampla e detalhada. A literatura mostra que, apesar da grande aplicabilidade desse exame, existem algumas deficiências como efeitos de teto e pouca sensibilidade para o comprometimento cognitivo leve ${ }^{27}$. Nesse sentido, a aplicação de vários testes permitirá que se avalie adequadamente, do ponto de vista tanto qualitativo como quantitativo, o estado cognitivo de cada paciente, o que contribuirá tanto para estabelecer o estado atual quanto para auxiliar o diagnóstico diferencial e também fazer um acompanhamento mais preciso da evolução do estado cognitivo. Quando existir a necessidade de aprofundar a avaliação, é indispensável a atuação de profissionais especializados.

O presente estudo possui algumas limitações que devem ser consideradas. Uma delas refere-se ao delineamento transversal que não permite verificar a relação causal entre as variáveis. O fato de a seleção da amostra ter sido realizada por conveniência também constitui uma limitação, já que, por não se tratar de uma amostra de base populacional, não se pode generalizar os resultados desta pesquisa. Outra limi- tação refere-se ao fato de que não foram realizadas análises do poder discriminativo do MEEM nos pacientes com AVC, o que pode ser abordado em estudos futuros. Neste estudo não foi abordada a relação entre os déficits cognitivos e a localização das lesões cerebrais específicas, em virtude de os exames de neuroimagem terem sido realizados em diferentes hospitais, não permitindo a padronização dos laudos médicos. Além disso, seria importante analisar a influência do lado da lesão cerebral no déficit cognitivo desses pacientes.

\section{CONCLUSÃO}

Os resultados apontaram que, por meio do rastreio positivo de déficit cognitivo, foram encontrados pontos de corte associados ao comprometimento neurológico, necessitando também serem ajustados pela idade e escolaridade, sugerindo que essas associações sejam preferencialmente levadas em consideração na avaliação e planificação da reabilitação neuropsicológica dos pacientes com AVC.

\section{CONTRIBUIÇÕES INDIVIDUAIS}

Todos os autores contribuíram significativamente na concepção e desenho do estudo, na análise e interpretação dos dados, na elaboração do artigo e na aprovação da versão final a ser publicada.

\section{CONFLITOS DE INTERESSE}

Não há conflitos de interesse a serem declarados.

\section{AGRADECIMENTOS}

Ao Conselho Nacional de Desenvolvimento Científico e Tecnológico (CNPq) (processo no 409797/2006-5), pelo apoio financeiro, e a todos os participantes do Programa de Diagnóstico e Intervenção no Acidente Vascular Cerebral (PRODIAVC).

\section{REFERÊNCIAS}

1. Nys GM, van Zandvoort MJ, de Kort PL, Jansen BP, de Haan EH, Kappelle LJ. Cognitive disorders in acute stroke: prevalence and clinical determinants. Cerebrovasc Dis. 2007;23(56):408-16.

2. Oneş K, Yalçinkaya EY, Toklu BC, Cağlar N. Effects of age, gender, and cognitive, functional and motor status on functional outcomes of stroke rehabilitation. NeuroRehabilitation. 2009;25(4):241-9.

3. Narasimhalu K, Ang S, De Silva DA, Wong MC, Chang HM, Chia KS, et al. The prognostic effects of poststroke cognitive impairment no dementia and domain-specific cognitive impairments in nondisabled ischemic stroke patients. Stroke. 2011;42(4):883-8. 
4. Milinavičienè E, Rastenytè D Kriščiünas A. Effectiveness of the second-stage rehabilitation in stroke patients with cognitive impairment. Medicina (Kaunas). 2011;47(9):486-93.

5. Patel M, Coshall C, Rudd A, Wolfe C. Natural history of cognitive impairment after stroke and factors associated with its recovery. Clin Rehabil. 2003;17(2):158-66.

6. del Ser T, Barba R, Morin MM, Domingo J, Cemillan C, Pondal M, et al. Evolution of cognitive impairment after stroke and risk factors for delayed progression. Stroke. 2005;36(12):2670-5

7. Pantoni L, Philip G. Advances in vascular cognitive impairment 2010. Stroke. 2011;42:291-3.

8. Serrano S, Domingo J, Rodríguez-Garcia E, Castro MD, del Ser T. Frequency of cognitive impairment without dementia in patients with stroke: a two-year follow-up study. Stroke. 2007;38(1):105-10

9. Pendlebury ST, Cuthbertson FC, Welch SJ, Mehta Z, Rothwell PM. Under estimation of cognitive impairment by Mini-Mental State Examination versus the Montreal Cognitive Assessment in patients with transient ischemic attack and stroke: a population-based study. Stroke. 2010;41:1290-3.

10. Bour A, Rasquin S, Boreas A, Limburg M, Verhey F. How predictive is the MMSE for cognitive performance after stroke? J Neurol. 2010;257:630-7.

11. Gottesman RF, Hillis AE. Predictors and assessment of cognitive dysfunction resulting from ischaemic stroke. Lancet Neurol. 2010;9(9):895-905.

12. Murden RA, McRae TD, Kaner S, Bucknam ME. Mini-Mental State Exam scores vary with education in blacks and whites. J Am Geriatr Soc. 1991;39:149-55.

13. Almeida OP. Miniexame do Estado Mental e o diagnóstico de demência no Brasil. Arq Neuropsiquiatr. 1998:56:605-12.

14. Caneda MAG, Fernandes JG, Almeida AG, Mugnol FE. Confiabilidade de escalas de comprometimento neurológico em pacientes com acidente vascular cerebral. Arq Neuropsiquiatr. 2006;64:690-7.

15. Klimkowicz-Mrowiec A, Dziedzic T, Słowik A, Szczudlik A. Predictors of poststroke dementia: results of a hospital-based study in Poland. Dement Geriatr Cogn Disord. 2006;21(5-6):328-34.

16. Rasquin SM, Verhey FR, van Oostenbrugge RJ, Lousberg R, Lodder J. Demographic and CT scan features related to cognitive impairment in the first year after stroke. J Neurol Neurosurg Psychiatry. 2004;75(11):1562-7.
17. Laks J, Batista EM, Guilherme ER, Contino AL, Faria ME, Figueira I, et al. Mini-mental state examination in community-dwelling elderly: preliminary data from Santo Antonio de Padua, Rio de Janeiro, Brazil. Arq Neuropsiquiatr. 2003;61(3B):782-5.

18. Brucki SM, Nitrini R, Caramelli P, Bertolucci PH, Okamoto IH. Sugestões para 0 uso do Miniexame do Estado Mental no Brasil. Arq Neuropsiquiatr. 2003;61(3-B):777-81.

19. Appelros P, Karlsson GM, Hennerdal S. Anosognosia versus unilateral neglect. Coexistence and their relations to age, stroke severity, lesion site and cognition. Eur J Neurol. 2007;14(1):54-9.

20. Gottesman RF, Kleinman JT, Davis C, Heidler-Gary J, Newhart M, Kannan V, et al. Unilateral neglect is more severe and common in older patients with right hemispheric stroke. Neurology. 2008;71(18):1439-44.

21. Engelter ST, Gostynski M, Papa S, Frei M, Born C, Ajdacic-Gross V, et al. Epidemiology of aphasia attributable to first ischemic stroke: incidence, severity, fluency, etiology, and thrombolysis. Stroke. 2006;37(6):1379-84.

22. Wright RM, Roumani YF, Boudreau R, Newman AB, Ruby CM, Studenski SA, et al. Effect of central nervous system medication use on decline in cognition in community-dwelling older adults: findings from the Health, Aging And Body Composition Study. J Am Geriatr Soc. 2009;57(2):243-50

23. Nys GM, van Zandvoort MJ, de Kort PL, van der Worp HB, Jansen BP, Algra A, et al. The prognostic value of domain-specific cognitive abilities in acute first-ever stroke. Neurology. 2005;64(5):821-7.

24. Leśniak M, Bak T, Czepiel W, Seniów J, Członkowska A. Frequency and prognostic value of cognitive disorders in stroke patients. Dement Geriatr Cogn Disord. 2008;26(4):356-63.

25. Pendlebury ST, Rothwell PM. Prevalence, incidence, and factors associated with prestroke and post-stroke dementia: a systematic review and meta-analysis. Lancet Neurol. 2009;8(11):1006-18.

26. Oliveira KCV, Barros ALS, Souza GFM. Miniexame do Estado Mental (MEEM) e (linical Dementia Rating (CDR) em idosos com doença de Alzheimer. Rev Neurocienc. 2008;16(2):101-6.

27. Srinivasan $S$. The concise cognitive test for dementia screening: reliability and effects of demographic variables as compared to the mini mental state examination. Neurol India. 2010;58(4):702-7. 